\title{
Dual-Polarized On-Chip Antenna for 300 GHz Full-Duplex Communication System
}

\author{
Linyan Guo, ${ }^{1,2}$ Ming Deng, ${ }^{1,2}$ Qisheng Zhang, ${ }^{1,2}$ Xinyue Zhang, ${ }^{2}$ and Zhenzhong Yuan² \\ ${ }^{1}$ Key Laboratory of Geo-Detection, China University of Geosciences, Ministry of Education, Beijing 100083, China \\ ${ }^{2}$ China University of Geosciences, Beijing 100083, China \\ Correspondence should be addressed to Ming Deng; dengming@cugb.edu.cn
}

Received 12 February 2017; Revised 2 June 2017; Accepted 7 June 2017; Published 9 July 2017

Academic Editor: Kerim Guney

Copyright (c) 2017 Linyan Guo et al. This is an open access article distributed under the Creative Commons Attribution License, which permits unrestricted use, distribution, and reproduction in any medium, provided the original work is properly cited.

\begin{abstract}
This paper presents a novel design of compact orthogonally polarized on-chip antenna to realize $300 \mathrm{GHz}$ full-duplex communication system with high isolation. It consists of a dipole antenna for horizontal polarization and a disk-loaded monopole antenna for vertical polarization. They are in good cross-polarization state with more than $90 \mathrm{~dB}$ of self-interference suppression and then can be used to achieve good isolation between transmitting and receiving antennas. In addition, two dual-polarized antennas have been adopted in two separated transceivers to study their isolation performance. Furthermore, this compact antenna only occupies an active area of $390 \mu \mathrm{m} \times 300 \mu \mathrm{m} \times 78 \mu \mathrm{m}$ and can be used for multiple-input multiple-output application as well.
\end{abstract}

\section{Introduction}

Full-duplex systems have attracted a lot of interest for allowing a radio node to transmit and receive signals simultaneously at the same frequency. They can potentially double the data rates achieved by half-duplex systems. However, selfinterference (SI) existing in full-duplex systems decreases the obtainable signal-to-interference ratio and makes them difficult to achieve in practice. Therefore, it is very important to suppress the SI at the receiver that is caused by coupling from its own transmitter. In earlier works, there are many ways to reduce the SI level below the receiver's noise floor, such as active cancellation, passive suppression, and a combination of them [1-3]. A majority of the overall SI suppression is due to passive suppression, that is, isolation between transmitting and receiving antennas. In a general way, there are three key mechanisms in the antenna domain. They are the directional isolation, absorptive shielding, and cross-polarization [4-7].

In these techniques, cross-polarization is one of the most common ways by using orthogonally polarized antennas. While the dual-polarized antenna favorably addresses the cross-polarization requirement, there are still some aspects that need be addressed to make the antenna fully suitable for future full-duplex communication. For example, the polarization isolation of orthogonally polarized antennas must be maximized. This is instrumental for the node to transmit signal with one polarization mode and receive it with an orthogonal polarization mode. However, in previous works, most traditional dual-polarized antennas achieve two polarization instances which are both parallel to the dielectric plane [8-10]. It is more suitable for them to be used between terminals on stacked boards. But, in some cases, terminals are placed transversely. Hence, in this paper, dual-polarized onchip antenna has been designed to realize a horizontal polarization along substrate surface and a vertical polarization perpendicular to substrate surface. It will be much suitable for two boards placed in side-by-side working situation with fullduplex communication. And this will make the full-duplex systems have a wider application prospect.

This paper is organized as follows. After introducing the design of dual-polarized on-chip antenna with high polarization isolation in Section 2, Section 3 studied the propagation property of two full-duplex nodes equipped with this dual-polarized antenna. Section 4 finally concludes this paper.

\section{Dual-Polarized Antenna}

The proposed dual-polarized antenna consists of a horizontally polarized dipole antenna and a vertically polarized 


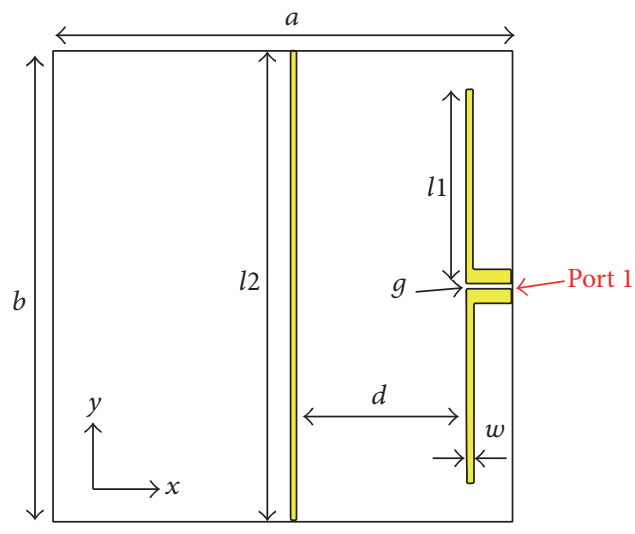

(a)

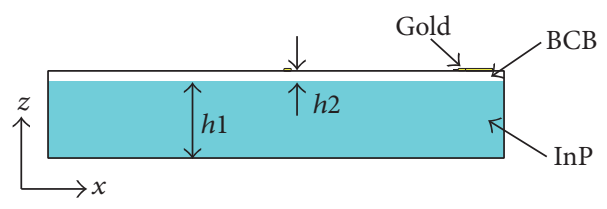

(b)

FIGURE 1: Schematic diagram of the dipole antenna. (a) Top view of antenna structure. (b) Side view of structure with different layers.

monopole antenna. It has two feeding ports: one is a balanced port to feed dipole antenna and the other one is a coaxial port to feed monopole antenna. In general, on-chip antennas are fed by small wafer probes. However, the characterization of these antennas cannot use probe-fed methods, as the probes are much larger than the antenna structures. Hence, the on-chip antenna could directly connect front integrated circuit after matching their complex impedances conjugately instead of a matching network [11]. The operations of separate antenna components have been simulated and optimized by a transient solver in CST Microwave Studio software. Since it is hard to fabricate and do experiment at this frequency, simulation results by HFSS are provided for comparison. In this work, in order to integrate this antenna with full-duplex modulator circuit, the on-chip antenna design is deposited on a benzocyclobutene (BCB) membrane and then an InP substrate, which is the same as $[12,13]$. At $300 \mathrm{GHz}$, the permittivity of InP substrate is 12.5 with loss tangent 0.002 , while the BCB substrate has a permittivity of 2.5 and loss tangent 0.005 .

2.1. Horizontally Polarized Dipole Antenna. Firstly, we simulated and optimized the performance of the horizontally polarized dipole antenna. Its geometry is sketched in Figure 1. It consists of a dipole antenna and a parasitic reflector which are placed on top of the $\mathrm{BCB}$ membrane. Whole sizes of the $\mathrm{InP}$ and $\mathrm{BCB}$ substrate in $x$ - and $y$-direction are $a=b=$ $300 \mu \mathrm{m}$. The InP substrate has a thickness $h 1=50 \mu \mathrm{m}$ and the BCB membrane has $h 2=6 \mu \mathrm{m}$. In simulation, metal of the dipole and reflector is gold with conductivity of $\sigma=$ $4.561 \times 10^{7} \mathrm{~S} / \mathrm{m}$ and thickness of $2 \mu \mathrm{m}$. Two dipole arms have been fed by a balanced feedline with length $25 \mu \mathrm{m}$ and width $10 \mu \mathrm{m}$ where a balanced port is fed here. Each dipole arm has identical length $l 1=125 \mu \mathrm{m}$ and width $w=5 \mu \mathrm{m}$. There is also a $g=2 \mu \mathrm{m}$ gap between two arms. We put the dipole arms $25 \mu \mathrm{m}$ away from right edge of the substrate. It is because the on-chip antenna gain is very poor when the dipole is mounted on the center of the chip, due to the lossy substrate and the close proximity of the radiation angle to the horizon [14]. Then a gold reflector was designed for reflecting electromagnetic wave to its right side with the purpose of achieving high directivity and increased antenna gain. It has a length $l 2=300 \mu \mathrm{m}$ and the same width with dipole arms $w=5 \mu \mathrm{m}$. In addition, distance between the dipole and reflector is $d=110 \mu \mathrm{m}$ which is approximately $\lambda / 4$. So the whole size of the dipole antenna is $300 \mu \mathrm{m} \times 300 \mu \mathrm{m} \times 58 \mu \mathrm{m}$.

Figure 2(a) shows the simulated magnitude of S11 parameter of the proposed dipole antenna by CST and HFSS. These curves in this figure have similar results and this means the CST results are acceptable. The dipole antenna has a bandwidth of $36.55 \mathrm{GHz}$ (from $291.45 \mathrm{GHz}$ to $328.0 \mathrm{GHz}$ ). Since two dipole arms are placed along $y$-axis and parallel to the surface of $\mathrm{BCB}$ and InP substrates, this dipole antenna is a horizontally polarized antenna. It can also be verified by $E$-field magnitude detected by a probe $5000 \mu \mathrm{m}$ away from the antenna on $x$-axes (not shown here). By detecting the $x$-, $y$-, and $z$-component of $E$-field magnitude, $y$-component is about $100 \mathrm{~dB}$ higher than $x$ - and $z$-component. Hence, this antenna is a horizontally polarized ( $y$-polarized) antenna. Figure 2(b) illustrates E-plane of the dipole antenna at $300 \mathrm{GHz}$. 2D E-plane of the proposed dipole antenna can be achieved by setting $\theta=90^{\circ}$. Since most energy points are at the direction of $\phi=0^{\circ}$, it means that the dipole antenna mainly radiates to its right side. $H$-plane in Figure 2(c) also shows that this antenna radiates to the right side. In addition, the simulated maximum gain of this dipole antenna is $4.91 \mathrm{dBi}$.

2.2. Vertically Polarized Monopole Antenna. Figure 3 shows the geometry of vertically polarized monopole antenna. Since its radiation arm travels through the substrate, it is a vertically polarized antenna. The $\mathrm{BCB}$ and $\mathrm{InP}$ substrates have the same size as that in horizontally polarized dipole antenna. In Figure 3(a), an annular gold ground is located on the backside of InP substrate with height $h g=20 \mu \mathrm{m}$, outer radius $r 1=98 \mu \mathrm{m}$, and inner radius $r 2=30 \mu \mathrm{m}$. A solid cylinder radiation travels through substrates and the annular ground with a radius of $r 3=13 \mu \mathrm{m}$ and height $h=78 \mu \mathrm{m}$. Then a gold disk is added on the top of the cylinder radiation in order to lengthen the monopole's electrical length (shown in Figure 3(b)). This gold disk has a thickness $2 \mu \mathrm{m}$ and radius $r 4=40 \mu \mathrm{m}$. In Figure 3(c), another parasitic annular ring has 


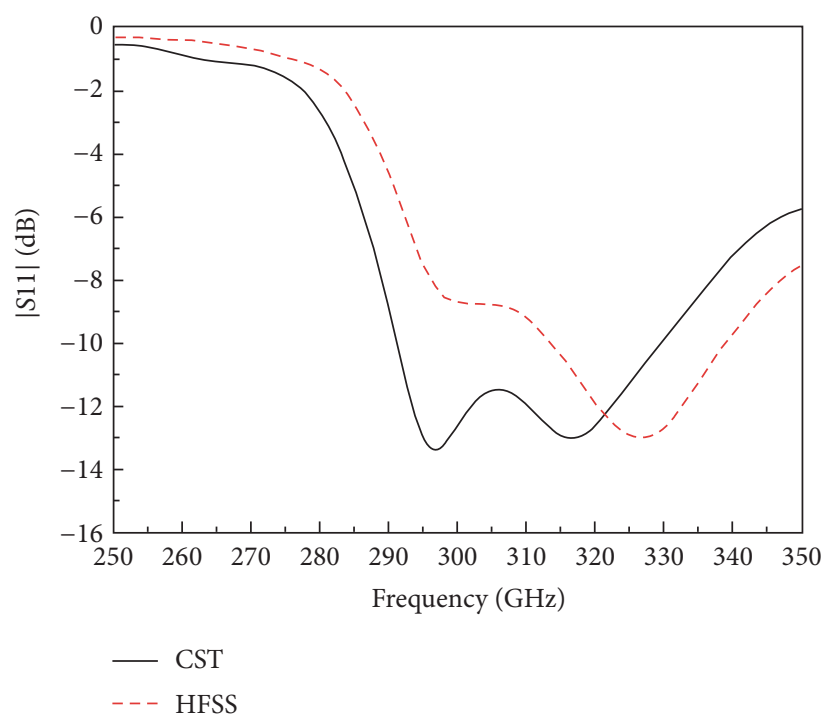

(a)

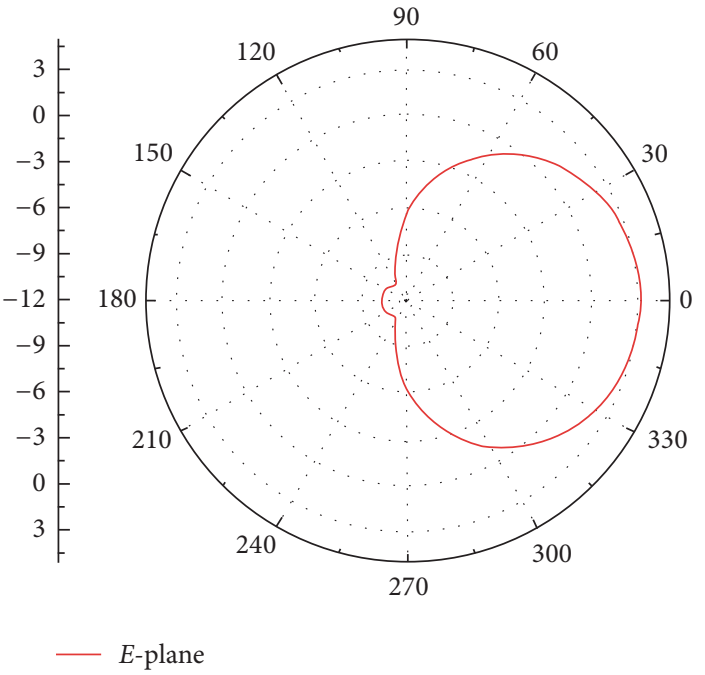

(b)

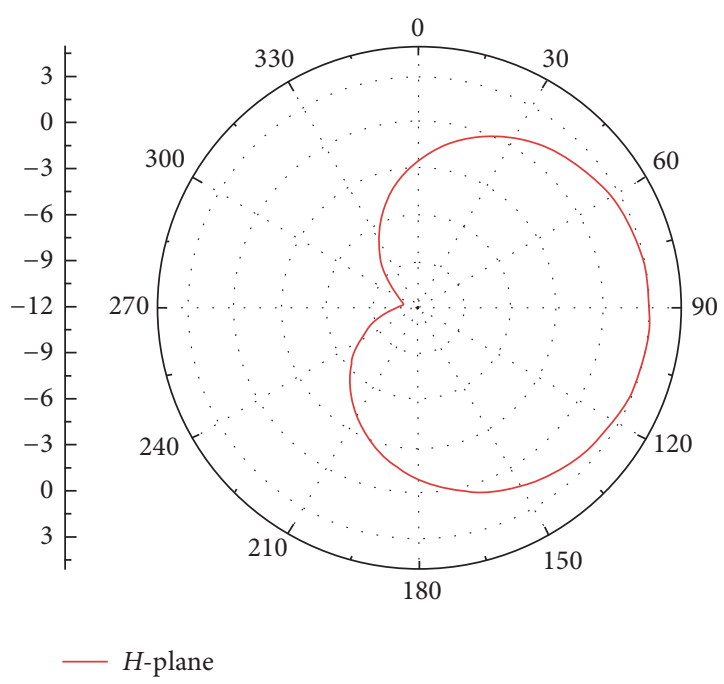

(c)

Figure 2: (a) Magnitude of $S 11$ in dB of the dipole antenna. (b) E-plane of the dipole antenna at $300 \mathrm{GHz}$ with $\theta=90^{\circ}$. (c) $H$-plane of the dipole antenna at $300 \mathrm{GHz}$ with $\phi=0^{\circ}$.

been added on the top of $\mathrm{BCB}$ substrate and located outside of the gold disk. It has an outer radius $r 5=98 \mu \mathrm{m}$ and inner radius $r 6=50 \mu \mathrm{m}$. In addition, four slender columns encircle the monopole and connect the top annular ring and ground. It can be seen in Figures 3(d) and 3(e). These four columns have the same dimensions with height $78 \mu \mathrm{m}$ and diameter $10 \mu \mathrm{m}$. After that, Figure 3(f) is the side view of the monopole antenna. Finally, in the case of coaxial feeding, this vertically polarized monopole antenna has a whole size of $300 \mu \mathrm{m} \times 300 \mu \mathrm{m} \times 78 \mu \mathrm{m}$.

Figure 4(a) shows the simulated magnitude of S11 parameter of the monopole antenna by CST and HFSS. It shows that these curves have similar results and this antenna has a narrow bandwidth of $6.07 \mathrm{GHz}$ (from $297.19 \mathrm{GHz}$ to $303.26 \mathrm{GHz}$ ). Figures $4(\mathrm{~b})$ and $4(\mathrm{c})$ illustrate its $E$-plane and
$H$-plane at $300 \mathrm{GHz}$. Figures 4(b) and 4(c) illustrate its $E$ plane and $H$-plane at $300 \mathrm{GHz}$ and show that this antenna is an omnidirectional antenna. This antenna radiates to the whole $x-y$ plane and has a maximum simulated gain of $1.72 \mathrm{dBi}$. This characteristic can also be verified by $E$-field magnitude detected by a probe placed away from the antenna on $x$-axes (not shown here). By detecting $x$-, $y$-, and $z$ component of $E$-field magnitude, $z$-component is the highest one and plays a dominant role when compared with the other two components. Hence, this monopole antenna is generally a vertically polarized ( $z$-polarized) antenna.

2.3. Dual-Polarized Antenna. Figure 5 shows the top view and side view of the dual-polarized antenna. Since the dipole antenna and monopole antenna are orthogonally polarized, 


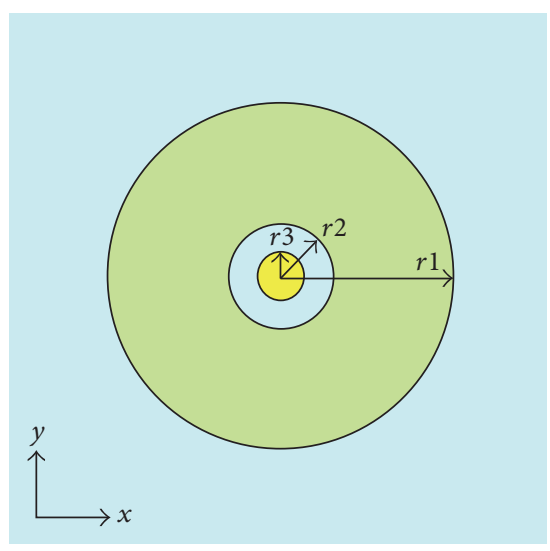

(a)

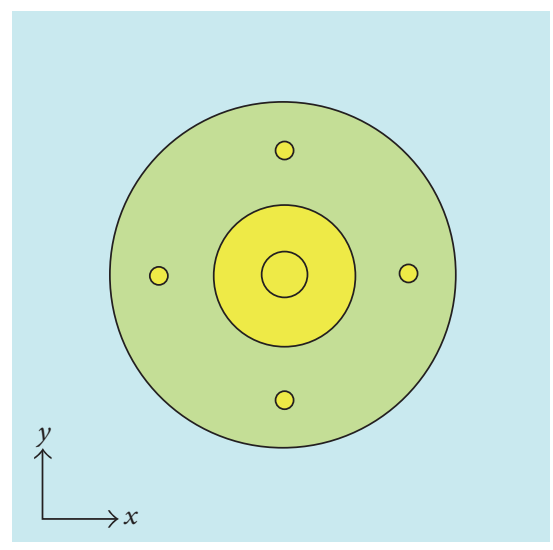

(d)

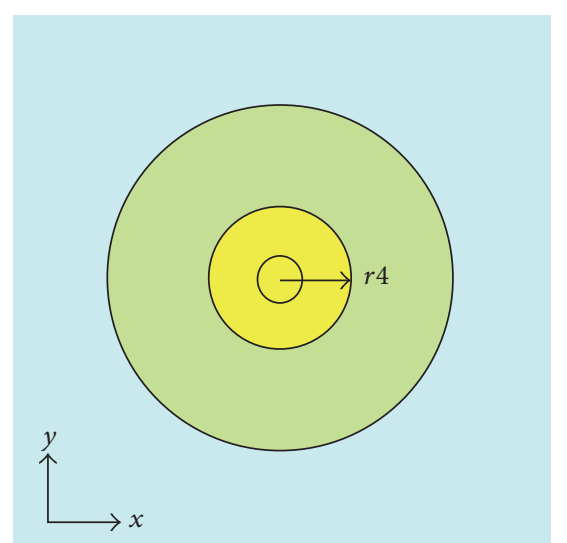

(b)

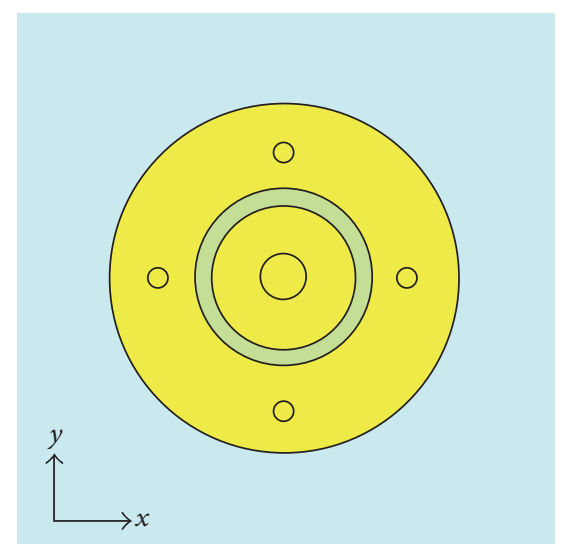

(e)

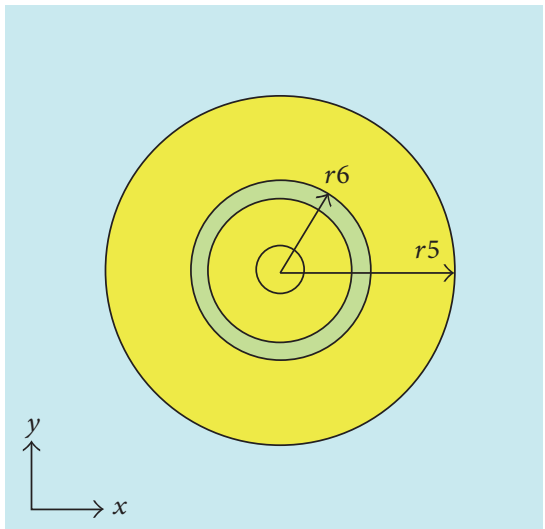

(c)

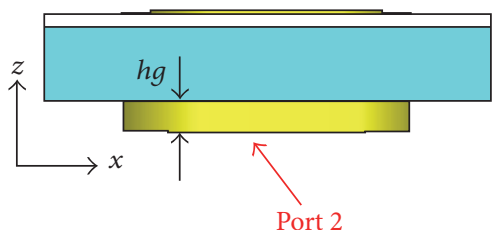

(f)

FIGURE 3: Schematic diagram of the vertically polarized monopole antenna.

they can work together with low cross coupling between each other. At the same time, they can also be put as close as possible. Hence, position of the dipole antenna and monopole antenna has been modified to achieve a compact size $390 \mu \mathrm{m} \times 300 \mu \mathrm{m} \times 78 \mu \mathrm{m}$. The dipole antenna still has a $25 \mu \mathrm{m}$ distance from the right edge of substrate, while the monopole has only a $12 \mu \mathrm{m}$ distance from the left edge. Consequently, distance between the dipole antenna and monopole antenna is only $37 \mu \mathrm{m}$, which is much smaller than antenna array's distance requirement $\lambda / 2$.

Figure 6 shows the simulated magnitude of $S$-parameter of two antenna components. In this figure, under the influence of monopole antenna, $|S 11|$ of horizontally polarized dipole has a bandwidth of $22.81 \mathrm{GHz}$ (from $291.45 \mathrm{GHz}$ to $314.26 \mathrm{GHz}$ ). It has a narrower bandwidth than its original model. $|S 22|$ is the magnitude of S-parameter of vertically polarized monopole. It has a bandwidth of $5.8 \mathrm{GHz}$ (from $295.6 \mathrm{GHz}$ to $301.4 \mathrm{GHz}$ ). In addition, $|S 12|$ is S-parameter when monopole transmits and dipole receives signal. Similarly, $|S 21|$ comes out when dipole transmits and monopole receives signal. In this figure, $|S 12|$ has the same value with $|S 21|$ within the whole research frequency band. In order to make a better comparison, this figure also provides $|S 12|$ by HFSS. It is $15 \mathrm{~dB}$ higher than the simulated results by CST. But both of them are about $90 \mathrm{~dB}$ lower than $|S 11|$ and $|S 22|$.
This means both the dipole and monopole have small impact on the other one. Therefore, this dual-polarized antenna can achieve SI suppression of $90 \mathrm{~dB}$ in the cross-polarization way. They have strong cross-polarization isolation and can work together even though they are put within a short distance.

A probe has also been placed at the same location. Similarly, $y$-component of dipole's $E$-field and $z$-component of monopole's $E$-field are the highest in their $E$-field magnitude. Hence, this antenna is a dual-polarized ( $y$ - and $z$-polarized) antenna. At the same time, the monopole and dipole are in orthogonal polarization states.

\section{Dual-Polarized Antenna in Transceivers for Full-Duplex System}

Different from most traditional dual-polarized antennas fed by one port, this dual-polarized antenna has two separate feeding ports. Figure 7 shows two identical nodes topology with the proposed dual-polarized antennas. In this sideby-side case, when horizontally polarized dipole in Node 1 (Antenna 1) works as a transmitter, another horizontally polarized antenna in Node 2 (Antenna 3) will work as a receiving antenna. At the same time, reverse link can also work effectively but in vertical polarization mode without any influence on the forward link. In other words, the monopole 


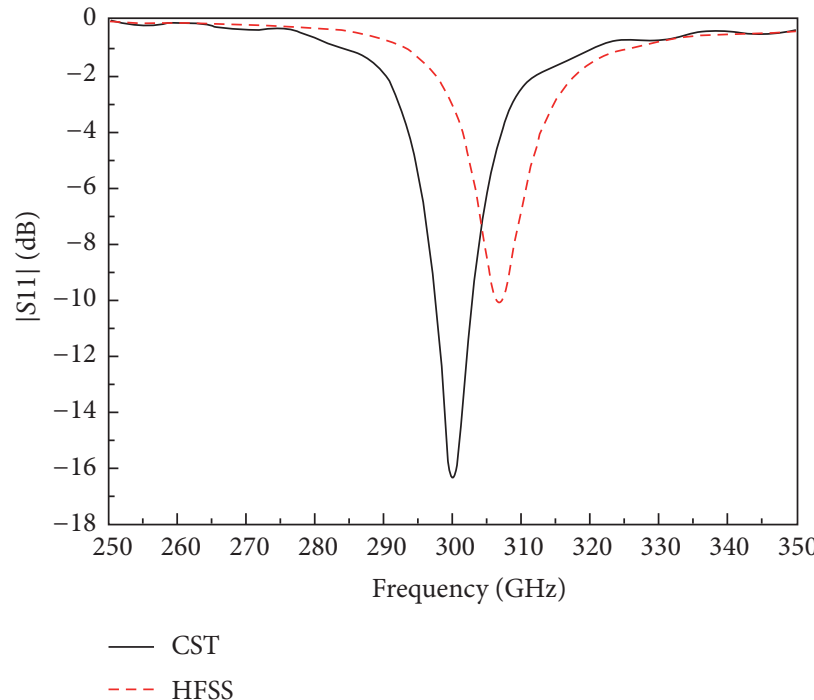

(a)

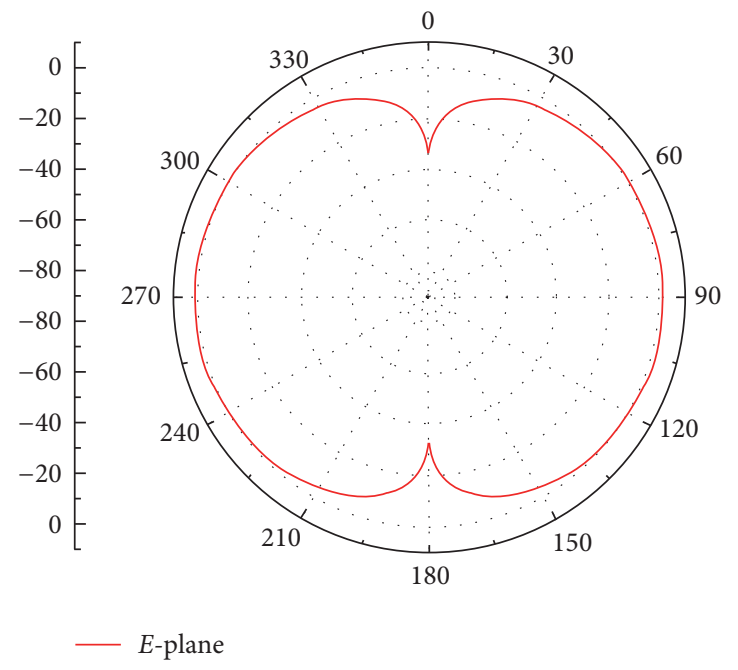

(b)

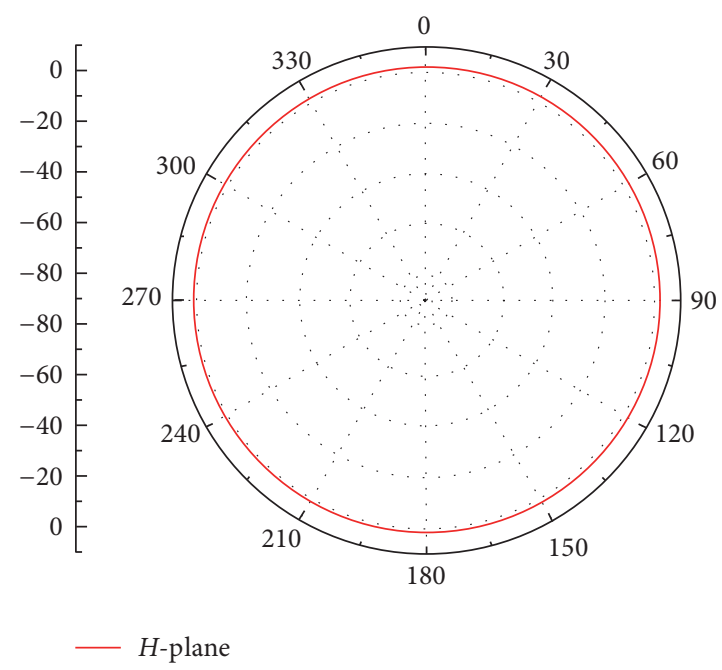

(c)

Figure 4: (a) Magnitude of $S 11$ in $\mathrm{dB}$ of the monopole antenna. (b) E-plane of the dipole antenna at $300 \mathrm{GHz}$ with $\phi=0^{\circ}$. (c) $H$-plane of the dipole antenna at $300 \mathrm{GHz}$ with $\theta=90^{\circ}$.

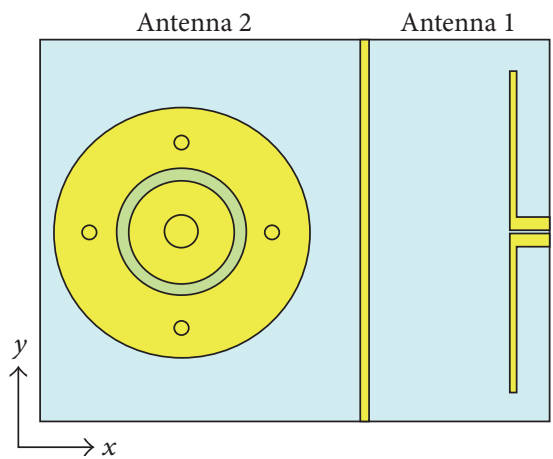

(a)

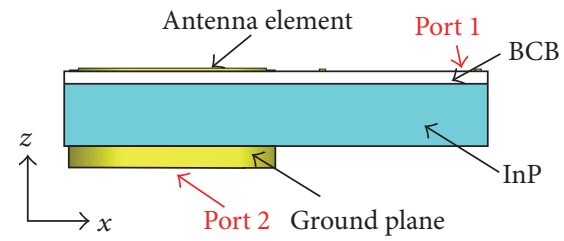

(b)

Figure 5: Dual-polarized antenna topology. (a) Top view. (b) Side view. 


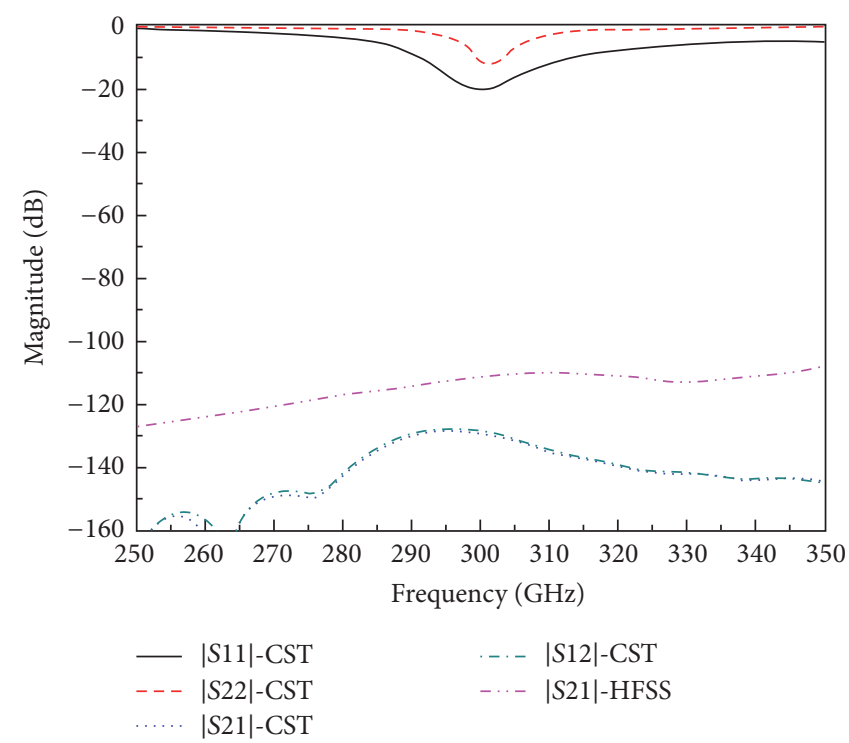

FIGURE 6: Simulated magnitude of $S$-parameter in $\mathrm{dB}$ of the dual-polarized antenna.
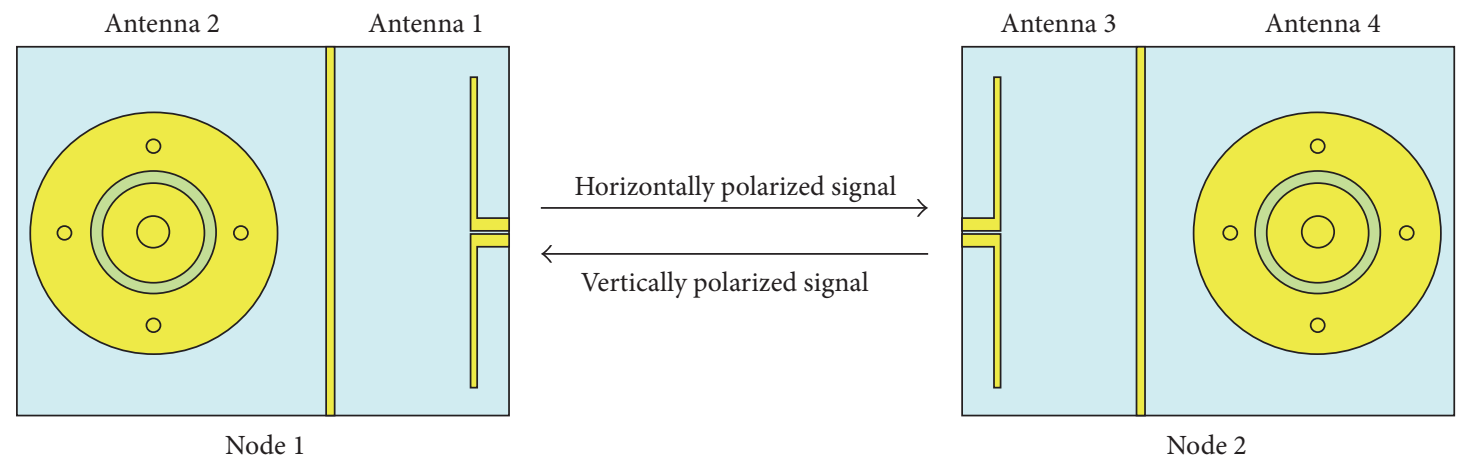

FIGURE 7: Schematic diagram of the $300 \mathrm{GHz}$ dual-polarized antenna in transceivers for full-duplex system. The simulation distance between two nodes is $5000 \mu \mathrm{m}$.

in Node 2 (Antenna 4) can transmit signal to monopole in Node 1 (Antenna 2) by the means of vertically polarized signals.

The performance of the dual-polarized antennas in fullduplex system is examined numerically. Figure 8(a) shows the isolation between Antenna 1 and antennas in Node 2. $|S 31|$ is S-parameter when Antenna 1 transmits and Antenna 3 receives signals. It can be seen that Antenna 3 has high cocoupling level with Antenna 1. It has the highest average receiving level of about $-43 \mathrm{~dB}$. $|S 41|$ is S-parameter when Antenna 1 transmits and Antenna 4 receives signals. In this case, the cross-polarized Antenna 4 has a lowest receiving level of $-160 \mathrm{~dB}$. Additionally, $|S 34|$ is the magnitude received by Antenna 3 when Antenna 4 transmits signals. It shows an average receiving level of $-124 \mathrm{~dB}$. That is a fairly low level and $81 \mathrm{~dB}$ lower than that received from Antenna 1. Hence, from the receiving level, the influence of Antenna 4 on Antenna 3 can be ignored when Antenna 3 receives signals from Antenna 1.
In the meantime, Antenna 4 of Node 2 can transmit data backward when Antenna 3 receives signals from Node 1. Figure $8(\mathrm{~b})$ shows that copolarized Antenna 2 has a receiving level of $-60 \mathrm{~dB}(|S 24|)$. It is about $60 \mathrm{~dB}$ higher than that received from Antenna 1. Hence, the influence of Antenna 1 is quite minimal. Meanwhile, the cross-polarized Antenna 1 $(|S 14|)$ has a relatively low receiving level of $-160 \mathrm{~dB}$.

\section{Conclusions}

We have proposed a dual-polarized on-chip antenna which can be used in $300 \mathrm{GHz}$ full-duplex communication system. Based on these results, we have proved that this orthogonally polarized antenna isolation technique is feasible. With the proposed technique, we obtain an average $90 \mathrm{~dB}$ SI isolation level with a compact antenna size. In future work, in order to completely suppress the SI of full-duplex nodes, we will investigate combining different isolation technologies including the proposed orthogonally polarized antenna technique. 


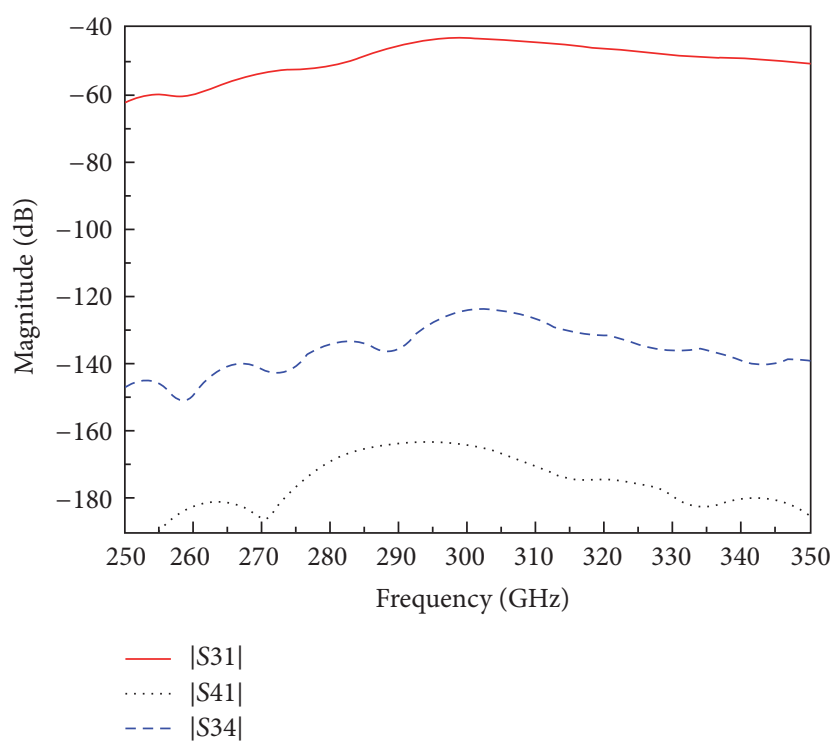

(a)

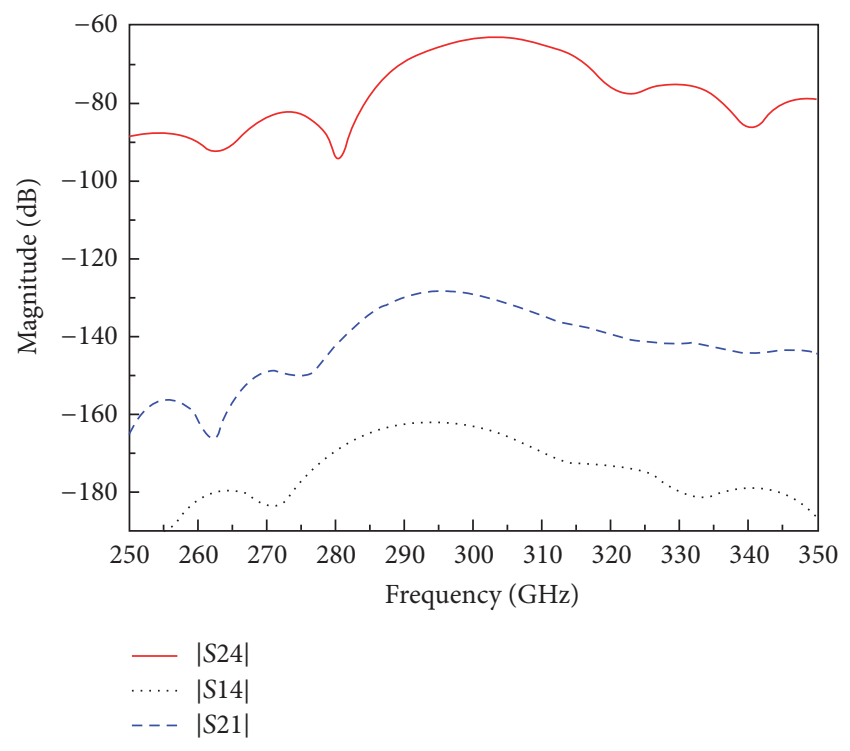

(b)

Figure 8: S-parameter of four antennas in Node 1 and Node 2. (a) Red straight line is cocoupling between Antenna 1 and Antenna 3. Black dot line is cross-polarized isolation between Antenna 1 and Antenna 4. Blue dash line is SI from Antenna 4 in Node 2. (b) Red straight line is cocoupling between Antenna 2 and Antenna 4. Black dot line is cross-polarized isolation between Antenna 1 and Antenna 4. Blue dash line is SI from Antenna 1 in Node 1.

\section{Conflicts of Interest}

The authors declare that they have no conflicts of interest.

\section{Acknowledgments}

The work was supported by the Open Fund (no. GDL1603) of Key Laboratory of Geo-Detection (China University of Geosciences, Beijing), Ministry of Education.

\section{References}

[1] B. Van Liempd, B. Debaillie, J. Craninckx et al., "RF self-interference cancellation for full-duplex," in Proceedings of the 9th International Conference on Cognitive Radio Oriented Wireless Networks (CROWNCOM'14), pp. 526-531, fin, June 2014.

[2] S. Hong, J. Brand, J. Choi II et al., "Applications of selfinterference cancellation in 5G and beyond," IEEE Communications Magazine, vol. 52, no. 2, pp. 114-121, 2014.

[3] D. Korpi, L. Anttila, V. Syrjälä, and M. Valkama, "Widely linear digital self-interference cancellation in direct-conversion full-duplex transceiver," IEEE Journal on Selected Areas in Communications, vol. 32, no. 9, pp. 1674-1687, 2014.

[4] E. Everett, A. Sahai, and A. Sabharwal, "Passive self-interference suppression for full-duplex infrastructure nodes," IEEE Transactions on Wireless Communications, vol. 13, no. 2, pp. 680-694, 2014.

[5] E. Foroozanfard, O. Franek, A. Tatomirescu, E. Tsakalaki, E. De Carvalho, and G. F. Pedersen, "Full-duplex MIMO system based on antenna cancellation technique," Electronics Letters, vol. 50, no. 16, pp. 1116-1117, 2014.

[6] M. Heino, S. N. Venkatasubramanian, C. Icheln, and K. Haneda, "Design of wavetraps for isolation improvement in compact inband full-duplex relay antennas," Institute of Electrical and Electronics Engineers. Transactions on Antennas and Propagation, vol. 64, no. 3, pp. 1061-1070, 2016.
[7] A. K. Khandani, "Methods for spatial multiplexing of wireless two-way channels," U.S. Patent 7817641 B1, 2010.

[8] O. Yurduseven, N. Llombart, A. Neto, and J. Baselmans, "A dual polarized antenna for $\mathrm{THz}$ space applications: antenna design and lens optimization," in Proceedings of the IEEE Antennas and Propagation Society International Symposium (APSURSI '14), pp. 191-192, IEEE, Memphis, Tenn, USA, July 2014.

[9] K. An, A. Chen, C. Yang, K. Zhang, and C. Li, "A dualpolarized microstrip antenna array with low cross-polarization for retrodirective antenna system," in Proceedings of the 2012 10th International Symposium on Antennas, Propagation and EM Theory (ISAPE'12), pp. 113-116, Xian, China, October 2012.

[10] D. S. Wang and C. H. Chan, "Novel terahertz dual-polarized frequency selective surface with high frequency selectivity," in Proceedings of the 2014 International Symposium on Antennas and Propagation (ISAP'14), pp. 207-208, Kaohsiung, Taiwan, December 2014.

[11] H. M. Cheema and A. Shamim, "The last barrier: On-chip antennas," IEEE Microwave Magazine, vol. 14, no. 1, pp. 79-91, 2013.

[12] H.-J. Song, J.-Y. Kim, K. Ajito, M. Yaita, and N. Kukutsu, "Fully integrated ASK receiver MMIC for terahertz communications at $300 \mathrm{GHz}$," IEEE Transactions on Terahertz Science and Technology, vol. 3, no. 4, pp. 445-452, 2013.

[13] H.-J. Song, J.-Y. Kim, K. Ajito, N. Kukutsu, and M. Yaita, “50$\mathrm{Gb} / \mathrm{s}$ Direct conversion QPSK modulator and demodulator MMICs for terahertz communications at $300 \mathrm{GHz}$," IEEE Transactions on Microwave Theory and Techniques, vol. 62, no. 3, pp. 600-609, 2014.

[14] F. Gutierrez Jr., S. Agarwal, K. Parrish, and T. S. Rappaport, "Onchip integrated antenna structures in CMOS for $60 \mathrm{GHz}$ WPAN systems," IEEE Journal on Selected Areas in Communications, vol. 27, no. 8, pp. 1367-1378, 2009. 


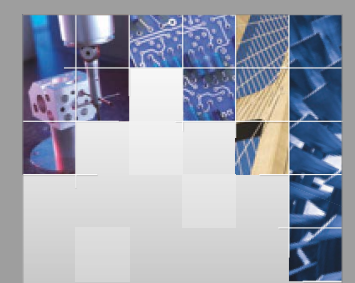

\section{Enfincering}
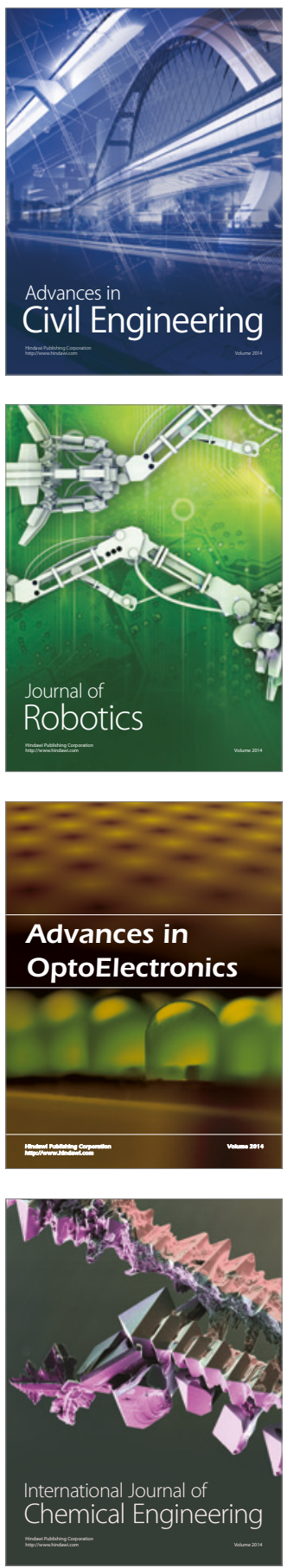

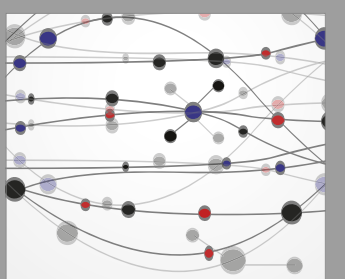

The Scientific World Journal

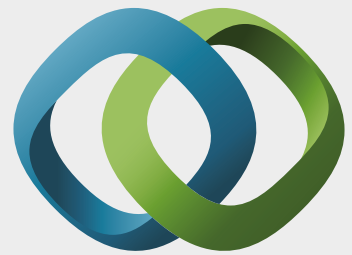

\section{Hindawi}

Submit your manuscripts at

https://www.hindawi.com
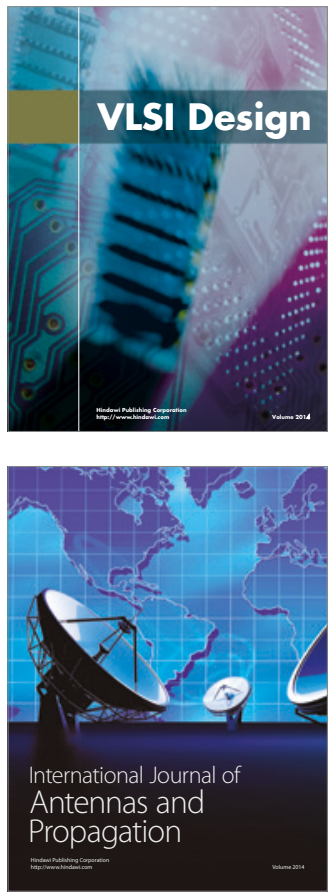

\section{Rotating}

Machinery
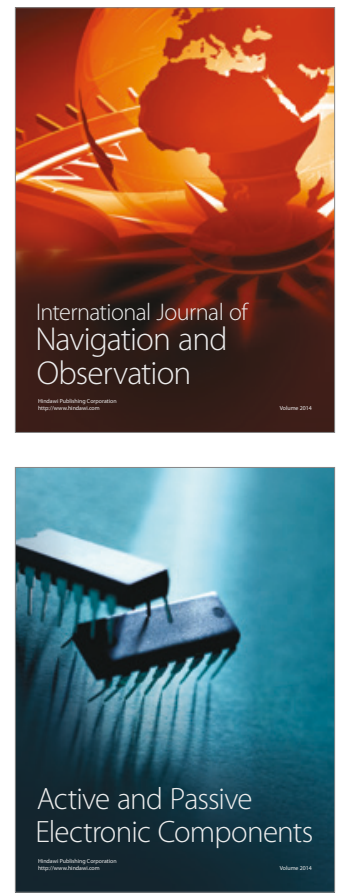
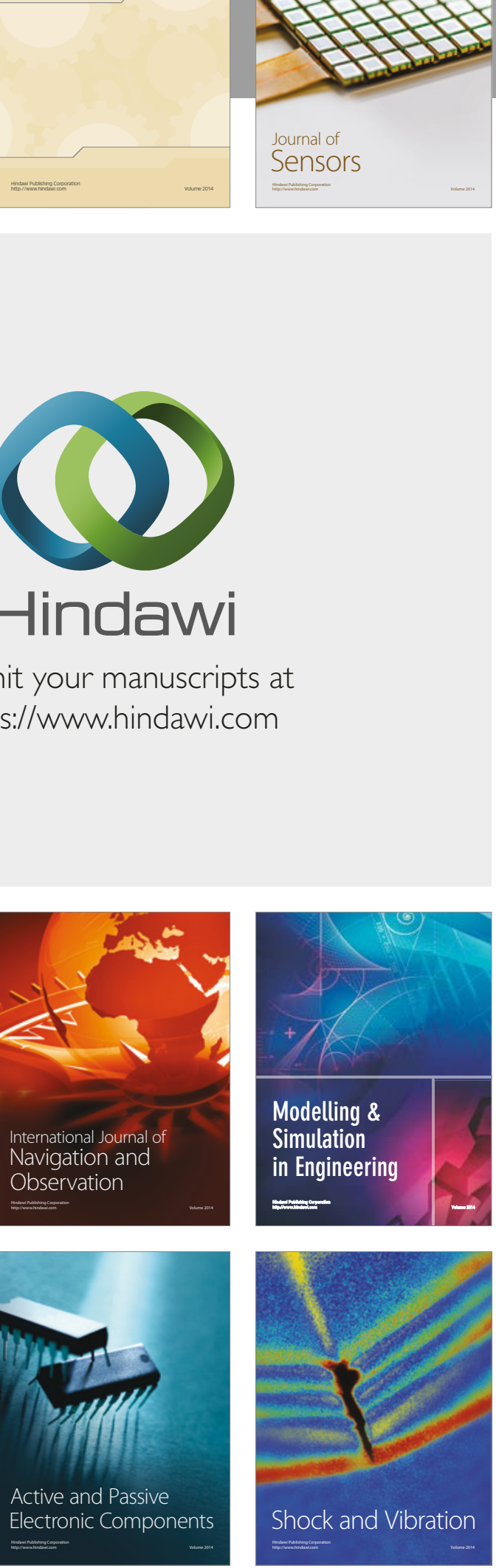
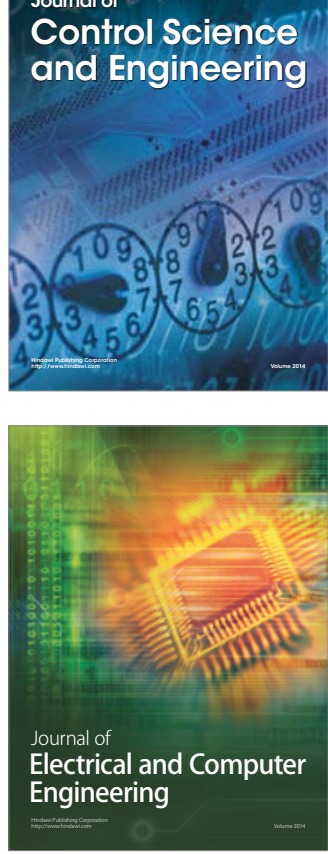

Distributed

Journal of

Control Science

and Engineering
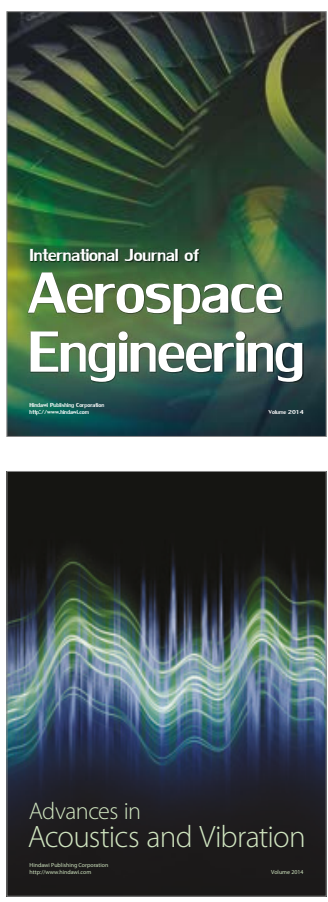

Sensor Networks 